\title{
Stephan Scharinger, Die Wunder des Pythagoras - Überlieferungen im Vergleich, Harrassowitz Verlag, Wiesbaden 2017, 262 pp., ISBN 978-3-447-10787-7
}

Stephan Scharinger - der Verfasser der vorliegenden Publikation - ist ein mit der LeopoldFranzens-Universität in Innsbruck verbundener Wissenschaftter. Die hier zu besprechende Studie, Die Wunder des Pythagoras - Überlieferungen im Vergleich, wurde als Dissertation im Wintersemester 2016/2017 verteidigt. Sie besteht aus acht Kapiteln, einem Literaturverzeichnis und einem Index. Das Hauptanliegen des Bandes ist die 'Wundertradition innerhalb der Pythagoraslegende sowie außerhalb im Vergleich mit anderen Wundertätern der Antike' zu untersuchen (S. 1). Das erste Kapitel stellt eine Einführung dar, in der Aufbau der Arbeit und Methodologie präsentiert wurde (S. 1-5). Was die Methodologie angeht, verwendet Scharinger „ein diskursanalytischer komparatistischer Ansatz“ (S. 3), indem er zu Beginn Texte über Pythagoras untersucht (der interne Vergleich), um Zusammenhänge zwischen verschiedenen Motiven festzustellen und darauffolgend „Gemeinsamkeiten und Unterschiede zwischen den Motiven der Pythagorasüberlieferung und jenen Topoi, die über andere Wundertäter berichtet werden, analysiert (der externe Vergleich)“'. Im nächsten Kapitel wurde die Quellenlage (S. 7-24), die sich auf die Gestalt des Pythagoras bezieht, präsentiert, wobei Scharinger weist darauf hin, dass die meisten Quellen über Pythagoras im Hellenismus oder in der Kaiserzeit entstanden. In diesem Teil des Buches wurde darüber hinaus nach Abhängigkeiten oder Brüchen in der Überlieferung gesucht. Im dritten Kapiltel setzt sich Scharinger sowohl mit dem curriculum vitae des Pythagoras, als auch mit den Pythagoreern auseinander (S. 27-73). In Betracht wurde beispielsweise das historische Umfeld gezogen, in dem der Philosoph lebte und wirkte, was einen Einfluß auf die pythagoreische Lehre ausübte - z.B. die Bewegung der Orphik. Danach, im vierten Kapitel, befasst sich Scharinger mit einer wesentlichen Frage, das heißt, was unter dem Begriff „Wunder“ zu verstehen ist (S. 73-93). Ein Wunder sei ,außergewöhnliche Handlung oder Tat, ein über-natürliches Phänomen oder Ereignis bzw. ein dem Herkömmlichen widersprechender Zustand“" (S. 75). Unter die Lupe wurden Wunder in ethnischen und den altindischen Religionen, in der griechisch-römischen Antike und im biblisch-frühchristlichen Bereich genommen. Zum Schluss dieses Kapitels wurden Wunder, die Pythagoras angehen, in fünf Kategorien eingeteilt: Heilwunder (z.B. Heilung und Beruhigung der Seele), Naturwunder (Macht über die unbelebte Natur, Tierwunder), Begleitwunder (z.B. Geburtslegende), Prophetie (prophetische Träume, Mantik und Vorhersagen), Wissen um die Seele (beispielsweise Unterweltsfahrt) (S. 90). Es soll des Weieteren darauf hingewiesen werden, dass auch verschiedene Begriffe, die Wundertaten bezeichnen, in diesem Kapitel erklärt wurden

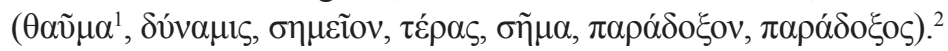

\footnotetext{
Im Text (S. 82) falsch $\theta \alpha$ ó $\mu \alpha$.

2 Vgl. z.B. S. $77-83$.
} 
Im nächsten Kapitel widmet sich der Verfasser dem internen Vergleich der Wunder in den bedeutungsvollsten Vitae des Pythagoras (S. 93-125). Es handelt sich hier um drei Werke, in denen das Leben und Handeln des Pythagoras dargestellt wurde - Diogenes Laertios (ca. 3. Jahrhundert nach Christi Geburt) und seine Leben und Lehre der

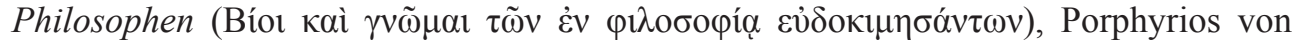
Tyros (3/4. Jahrundert n. Chr.) und Iamblichos von Chalkis (3/4. Jahrundert n. Chr.), der das Leben des Pythagoras im ersten Buch seines Werkes Über die pythagoreische Schule präsentierte. Aus dem von Scharinger durchgeführten internen Vergleich geht hervor, dass während Porphyrios und Iamblichos sehr ausgiebig über Wunder des Pythagoras berichten (z.B. Heilungen, Beherrschung der Natur) kommen Beschreibungen der pythagoreischen Wundertaten bei Diogenes Laertios seltener vor, was die Tabelle 2 (Die pythagoreischen Wundertaten bei Diogenes Laertios, Porphyrios und Iamblichos) äußerst deutlich veranschaulicht (S. 124-125). Im Leben und Lehre der Philosophen erfährt man beispielsweise, dass Pythagoras von seinen Schülern für den hyperboreischen Apollon gehalten worden sei. ${ }^{3}$ Pythagoras wurde darüber hinaus Reisen in den Orient (z.B. nach Ägypten) zugeschrieben, was auch in Bezug auf andere бópor (beispielsweise Solon) festzustellen ist. ${ }^{4}$ Im sechsten Kapitel (,Externer “ Vergleich; Die Wundertaten und ihr typologischer Kontext) beschäftigt sich Scharinger mit den Berichten über die Wundertaten des Pythagoras, die in verschiedenen Quellen präsent sind, vergleicht sie miteinander, „um sie anschließend in einen größeren Zusammenhang mit den Berichten über andere Gestalten aus Religion und Kult zu stellen“ (S. 127). In dieser Analyse spielt die Einteilung der Wunder aus dem vierten Kapitel eine bedeutsame Rolle. Der externe Vergleich, der im sechsten Kapitel durchgeführt wurde, scheint völlig gelungen zu sein. Scharinger macht uns deutlich, inwieweit Wundertaten, die Pythagoras zuzuschreiben sind, auch in anderen Quellen (nicht nur der griechisch-römischen Antike), Religionen (z.B. Schamanismus) und Kulturen auftauchen. Eine hilfsreiche Zusammenfassung der gesamten Untersuchung stellt die umfangreiche Tabelle 3 (Die pythagoreischen Wundertaten im vergleichenden Aspekt) dar, in der alle pythagoreischen Wunder mit dem Vergleichmaterial zusammengestellt wurden.

Zusammenfassend kann man feststellen, dass der vorliegende Band eine wichtige und anregende Studie ausmacht, die hoffentlich zu einer weiteren Disskusion über die Wundertaten in der antiken Welt und Literatur führen wird.

Jakub Kuciak (Jagellonen-Universität zu Krakau)

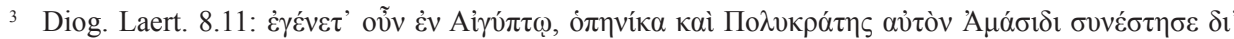
$\dot{\varepsilon} \pi 1 \sigma \tau \circ \lambda \tilde{\eta} \varsigma$.

4 Pythagoras gehörte zu den sogenannten Sieben Weisen; vgl. z.B. J. Engels, Die sieben Weisen - Leben, Lehren und Legenden, München 2010, 70-74; die Frage, wann die Liste der sieben Weisen entstand, vgl. z.B. D. Fehling, Die sieben Weisen und die frühgriechische Chronologie - eine traditionsgeschichtliche Studie, Bern-Frankfurt am Main-New York 1985. 